Available online at GSC Online Press Directory

GSC Biological and Pharmaceutical Sciences

e-ISSN: 2581-3250, CODEN (USA): GBPSC2

Journal homepage: https://www.gsconlinepress.com/journals/gscbps

(SHORT COMMUNICATION)

\title{
The effect of ICSI-related procedural timings and operators on the outcome
}

\author{
Yovich John L* \\ Medical Director PIVET Medical Centre Perth, Western Australia, Australia 6007. \\ Publication history: Received on 24 March 2020; revised on 29 March 2020; accepted on 31 March 2020
}

Article DOI: https://doi.org/10.30574/gscbps.2020.11.1.0075

\begin{abstract}
A recent publication from a private IVF facility in Rome reports surprising findings in respect to ICSI-related procedural timings. The GENERA group reported on ICSI outcomes with fresh autologous oocytes where the insemination process was conducted with fresh non-donor ejaculated sperm. With primary outcome being the cumulative delivery rate (CDR) and secondary outcome the mean blastulation rate, the group showed that the latter was significantly higher when oocyte denudation for ICSI was performed less than 38 hours from ovulation induction rather than their usual 38-42 hours. Furthermore, they concluded that CDR was not influenced by ICSI timings where oocyte denudation was conducted between 2 to 7 hours from oocyte retrieval. This report has very significant implications for the logistical management of embryology laboratories, but most IVF clinics will be skeptical for two reasons - firstly the GENERA researchers failed to cite any of the early pioneer work regarding IVF insemination times, conducted from various locations in the 1980's; all showing optimal outcomes when insemination was performed 5.5 to 7.5 hours post oocyte retrieval, matching around 42 hours post ovulation trigger. Secondly, the statistical analysis for their data was rather complex including multivariate logistic regression analyses which did not provide clearly conclusive results, certainly not sufficient to cause long-standing IVF operators to change practice. Within this era of evidence-based medicine, it is difficult to gain confidence in studies that appear to suggest nothing from the first 20 years of IVF was reliable.
\end{abstract}

Keywords: ICSI timings; Oocyte denudation; Egg stripping; Ovulation induction; Blastocyst development rate; Cumulative livebirth rate

\section{Introduction}

\subsection{Insemination timings}

I was very interested in the recent article from the GENERA group in Rome [1] as their data conflicts with that of the early pioneers regarding the optimal timing of insemination, and which is inconsistent with our current practice [2].

\subsection{Summary of GENERA study}

The GENERA group reported on intra-cytoplasmic sperm injection (ICSI) outcomes with fresh autologous oocytes where the insemination process was conducted with fresh non-donor ejaculated sperm. With primary outcome being the cumulative delivery rate (CDR) and secondary outcome the mean blastulation rate, the group showed that the latter was significantly higher when oocyte denudation for ICSI was performed less than 38 hours from ovulation induction rather than their usual 38-42 hours. Furthermore, they concluded that CDR was not influenced by ICSI timings where oocyte denudation was conducted between 2 to 7 hours from oocyte retrieval.

\footnotetext{
${ }^{*}$ Corresponding author: Yovich John L
} 


\section{Criticism of GENERA study}

As one who has explored in-vitro fertilization (IVF) from 1977 [3,4] and maintains an active Medical Directorship role today, I am critical of two negative trends apparent in the GENERA article.

\subsection{First Criticism - failure to acknowledge historical publications}

Firstly, and with due respect to the senior authors, it appears that the literature search was largely electronic, hence missing out on key references which have guided my IVF practices to this day. In particular an important study from the pioneer Monash IVF unit in Melbourne [5] showed the benefit of delaying insemination for up to 6.5 hours post oocyte pick-up (OPU). In its day, this significantly improved the embryo development outcomes and was confirmed in a subsequent study from Zagreb in Croatia the following year [6]. The latter study showed optimal embryo development in the group who had fertilization delayed 5.5 to 7.5 hours following OPU undertaken at 36 hours post-Ovulation Trigger with hCG. Following the success of gamete intrafallopian transfer (GIFT) treatments introduced in 1985 whereby sperm and oocytes are placed simultaneously into the fallopian tube, a further important study was conducted by the Queensland Fertility Group [7]. Although reasonable rates of fertilization could be achieved by 3 hours, optimal rates along with optimal pregnancy rates, were achieved after 5 hours, even as late as 13 hours. In the PIVET facility, our protocol favours 6 hours post-oocyte pick-up (OPU) for both IVF and ICSI, but I am sometimes in conflict with my embryologists who reduce this period for logistical reasons. This comes to my attention when poor fertilization outcomes or poor embryo development occurs; such cases being routinely analysed in detail by our clinico-laboratory group [8].

\subsection{Second criticism - complex, unconvincing statistical analysis}

The second point of criticism relates to the statistical evaluation of their data. As a sceptic of randomised controlled trial (RCT) studies, I do admire that "this is an observational study conducted at a private Italian IVF clinic" over a recent 2year period as this means that it represents a typical situation with which most IVF clinics, including PIVET, can identify. However, in keeping with modern trends, the data has been analysed "in generalized linear models and multivariate logistic regression" applying SPSS software. The Figures and Tables present a complex profile to cover for many potential confounders and which represents high-level statistical expertise which we can only trust is telling the accurate story. For those whose expertise rests with Chi-squared, t-test and analysis of variance (ANOVA) comparisons, the use of these more-advanced statistical methods can be bewildering. Furthermore, my understanding is that they can be fraught with incorrect conclusions if not skillfully applied and even experts of SPSS warn of its limitations in these areas [9].

\section{Conclusion}

Whilst not wishing to challenge the statistical methodologies of the GENERA group, I would simply question why the work from the entire 1980's was ignored; and how can the disparate findings be reconciled with the earlier studies? Furthermore, senior members of the IVF world would seek guidance on which studies should provide our lead? Managerial IVF clinicians and scientists will be in anticipation of the answers, as it may mean that late-day and evening insemination procedures established under current protocols, may be avoidable without compromising outcomes. This idea is certainly one which has appeal, but the GENERA article is not sufficiently convincing to cause long-standing IVF operators to change practice. Furthermore, within this era of evidence-based medicine, it is difficult to gain confidence in studies that appear to suggest nothing reported during the first 20 years of IVF was reliable.

\section{Compliance with ethical standards}

\section{Acknowledgments}

As Medical Director, I wish to acknowledge the support of my embryology staff headed by Laboratory Director Jason L Conceicao who is responsible for any adjustments to PIVET protocols and who defends the laboratory processes required for annual accreditation by the national regulatory body known as RTAC (the Reproductive Technology Accreditation Committee) under the auspices of the Fertility Society of Australia.

\section{Disclosure of conflict of interest}

This is a self-funded, sole-author publication and there are no conflicts of interest to declare. 


\section{References}

[1] Maggiulli R, Cimadomo D, Fabozzi G, Papini L, Dovere L, Ubaldi FM and Rienzi L. (2020). The effect of ICSI-related procedural timings and operators on the outcome. Hum Reprod, 1, 1-12.

[2] Yovich JL, Conceicao JL, Marjanovich N, Ye Y, Hinchliffe PM, Dhaliwal SS and Keane KN. (2018). An ICSI rate of $90 \%$ minimizes complete failed fertilization and provides satisfactory implantation rates without elevating fetal abnormalities. Reprod Biol, 18, 301-311.

[3] Yovich JL and Craft IL. (2018). Founding pioneers of IVF: Independent innovative researchers generating livebirths within 4 years of the first birth. Reprod Biol, 18, 317-323.

[4] Yovich JL. (2020). Founding pioneers of IVF Update: Independent innovative researchers generating livebirths within 4 years of the first birth. Reprod Biol, 20, 111-113.

[5] Trounson AO, Mohr LR, Wood C and Leeton JF. (1982). Effect of delayed insemination on in-vitro fertilization, culture and transfer of human embryos. J Reprod Fertil, 64(2), 285-294.

[6] Maćas E, Suchanek E, Grizelj V, Simunić V, Puharić I and Drobnjak P. (1983). The effect of delayed insemination on fertilization in vitro and the development of human embryos. Jugosl Gynekol Opstet, 23(5-6), 106-111.

[7] Harrison KL, Pope AK, Wilson LM, Cummins JM, Breen TM and Hennessey JF. (1988). Fertilization of human oocytes in relation to varying delay before insemination. Fertil Steril, 50(2), 294-297.

[8] Yovich JL. (2019). Monitoring the stimulated IVF cycle. In: How to Prepare the Egg and Embryo to Maximise IVF Success. Section II: Stimulation for IVF (Eds: Gabor T Kovacs, Anthony J Rutherford, David K Gardner). Cambridge University Press, Cambridge, 94-120.

[9] Field A. (2013). Discovering statistics using IBM SPSS Statistics. 4th edition. Sage publications, London, 916.

\section{How to cite this article}

Yovich JL. (2020). The effect of ICSI-related procedural timings and operators on the outcome. GSC Biological and Pharmaceutical Sciences, 11(1), 09-11. 